\title{
Antimalarials - are they effective and safe in rheumatic diseases?
}

\author{
Ewa Haładyj ${ }^{1}$, Mariusz Sikora ${ }^{2}$, Anna Felis-Giemza ${ }^{1}$, Marzena Olesińska $^{1}$ \\ ${ }^{1}$ Department of Connective Tissue Diseases, National Institute of Geriatrics, Rheumatology and Rehabilitation, Warsaw, Poland \\ 2Department of Dermatology, Medical University of Warsaw, Poland
}

\begin{abstract}
Antimalarial drugs (AD) are a group of widespread therapeutic agents in multiple rheumatic indications. Although the effect of AD is mild and extended in time, low toxicity is their appreciated value. This paper describes the current state of knowledge on the mechanism of action, use, toxicity and pleiotropic effects of $A D$ in the pharmacotherapy of autoimmune diseases.
\end{abstract}

Key words: antimalarials, hydroxychloroquine, chloroquine.

\section{Introduction}

Antimalarial drugs (AD) have a very long and interesting history. Soon it will be 400 years since the first use of quinine. According to legend, the Countess of Chinchon, wife of the Spanish viceroy of Peru, was cured of malaria in 1630 thanks to the powdered bark of a tree which was used widely by local Indians as an antipyretic. Although the story does not seem to be true, a hundred years later the Swedish naturalist Carl Linnaeus was inspired by it to name the "miracle tree" cinchona [1].

In fact, the healing properties of quinine bark were discovered by Spanish Jesuit missionaries who brought the bark and spread it throughout Europe. Today we know that an extract from the bark of the tree corresponds to a mixture of over 30 active alkaloids with antipyretic, anti-inflammatory, anti-malarial, and even myorelaxant and antiarrhythmic properties. The first separate substance from this group was quinine, which in 1820 was isolated by JB Caventou and PJ Pelletier. For more than 100 years the extraction of oil from the bark was the only way of obtaining the drug, until 1944, when the compound was obtained by chemical synthesis [1].

During World War II, the US government funded a special team of scientists and engineers who created more effective, less toxic and easier to manufacture antimalarials. The most promising substance was chloro- quine, which had already been synthesized in the Bayer laboratory in 1934, by Hans Andersaga, under the name Resochin. But then, it was concluded that the drug was too toxic and further research was stopped.

With World War II the first information on the potential efficacy of antimalarials in rheumatic diseases was revealed. American soldiers who fought in the Pacific region received antimalarials in prophylaxis. Reduction of the severity of lesions and symptoms of arthritis was observed [2]. It led to further research on the efficacy and application of AD for the treatment of autoimmune diseases.

Antimalarials currently have an established position in the rheumatic armamentarium. However, in the era of modern immunosuppressive drugs and biological therapy, the significant therapeutic potential of this group may be underestimated. However, evidence for the beneficial actions of AD still appears in the medical literature, despite their immunomodulatory effect. This paper describes the current state of knowledge on the mechanism of action, use, toxicity and pleiotropic effects of $A D$ in the pharmacotherapy of rheumatic diseases.

\section{Pharmacology of antimalarials}

Chloroquine (CQ) and hydroxychloroquine (HCQ) are widely used in the treatment of autoimmune diseases.

\section{Address for correspondence:}

Ewa Haładyj, Department of Connective Tissue Diseases, National Institute of Geriatrics, Rheumatology and Rehabilitation, 1 Spartańska St., 02-637 Warsaw, Poland, e-mail: ehaladyj@o2.pl

Submitted: 18.03.2018; Accepted: 9.05.2018 
These drugs have similar structure, pharmacokinetics, mechanism of action, indications and side effects. Major discrepancies between AD pharmacology can be found. This is due to different protocols adopted in individual studies, different periods of time and medication dosage. Individual pharmacokinetic parameters significantly affect the activity of the underlying disease or concomitant diseases, e.g. diabetes, and it should be taken into consideration in therapeutic decisions.

\section{Composition}

Chemically, two substances are derivatives of 4-aminoquinoline. Hydroxychloroquine was synthesized in 1946 by hydroxylation of the ethyl group in the CQ molecule. Antimalarial drugs have chiral construction and therefore exist as a racemic mixture of two enantiomers $\mathrm{S}(+)$ and $\mathrm{R}(-)$ with different pharmacological properties [3]. The first has lower plasma concentration, greater bound protein and shorter half-life due to rapid metabolism and excretion. In studies in mice enantiomer S (+) $\mathrm{CQ}$ is characterized by greater antimalarial efficacy and lower toxicity. Data on efficacy and toxicity of enantiomers in rheumatic diseases are lacking. In the future, mixtures replacing enantiomers by the active stereoisomers may allow the dose to be lowered with increased efficacy of treatment and reduction of the potential side effects.

\section{Absorption}

After oral administration, CQ and HCQ are rapidly and almost completely absorbed from the gastrointestinal tract. Antimalarial drugs taken with food (especially rich in fats or proteins) increases the absorption and bioavailability. Maximum serum concentration is reached after 3-6 hours [4].

Animalarial drugs moderately bind to plasma proteins in about $60 \%$ for CQ and 50\% for HCQ [5]. Part of the drug is also associated with platelets [6]. Since the number of thrombocytes can be increased in active inflammatory diseases, such as rheumatoid arthritis (RA), it can significantly affect the pharmacokinetics of AD.

\section{Distribution}

The volume of $A D$ distribution is very high and exceeds $100 \mathrm{~L} / \mathrm{kg}$, which indicates accumulation of the drug in the tissue compartment [4]. Whole blood concentration is several times higher than in plasma due to the accumulation in blood cells: erythrocytes, thrombocytes and primarily in leukocytes, which is related to the mechanism of action. The AD concentration in internal organs is up to 1000 times values observed in plasma.
Due to the high affinity of CQ and HCQ for melanin, the highest concentration of $A D$ is in the skin (epidermis values are much higher than in the dermis) and in the eye [6]. High AD concentrations were also found in the spleen, liver, kidney, lung, heart and muscle.

\section{Metabolism and excretion}

Antimalarials are slowly excreted from the body and their half-life ranges from 20-60 days for CQ and 40-50 days for HCQ [4]. Due to slow release of the tissue compartment, the drug can be detected in urine even up to several years after the end of therapy [4].

The major route of elimination of $A D$ and its metabolites is via the kidney. Therefore, in patients with renal failure the half-life of the drug is prolonged and they need appropriate dosage adjustment [4].

CQ is removed $40-70 \%$ unchanged by the kidneys with active secretion as the predominant mechanism. Active derivatives of CQ (monodesethylchloroquin and bisdesethylchloroquin) are products of liver metabolism of the remaining $25-40 \%$ of the drug, while a small amount (5-10\%) is excreted to the feces [7]. Interestingly, the cytochrome P450 system is involved in metabolism of HCQ to desethylchloroquine, desethylhydroxychloroquine and bisdesethylhydroxychloroquine.

\section{Dosage}

There is no well-defined dose-effect curve for CQ. Chloroquine in a dose of $250 \mathrm{mg} /$ day provides a stable concentration in the range $100-500 \mathrm{ng} / \mathrm{ml}$. The recommended dose of HCQ is 200-400 mg/day. The beginning of action of HCQ can be seen after 4-6 weeks, while full efficacy can be assessed after 3 to 6 months. In the study by Furst et al. [7], HCQ administration at 1200 mg/day for the first 6 weeks was associated with a clinical response in patients with RA, but the rate of side effects from the gastrointestinal tract was significantly higher. The relationship between the concentration and efficacy of HCQ in the treatment of RA and systemic lupus erythematosus (SLE) patients has been confirmed [8,9].

\section{Interactions}

HCQ may increase the bioavailability of metoprolol, by inhibition of its metabolism. A similar effect of $A D$ was confirmed for digitalis - concomitant use causes an increase in the serum levels with higher risk of toxicity.

CQ may reduce the bioavailability of methotrexate, which probably contributes to the reduction of its hepatotoxicity. However, in combination with other disease-modifying antirheumatic drugs (DMARDs) such as 
cyclosporine and penicillamine it leads to an increase in their blood concentration.

Other drugs may also affect the AD pharmacokinetics. Cimetidine reduced CQ clearance by more than $50 \%$ [4]. Theoretically, there is an opportunity of AD interaction with proton pump inhibitors (PPIs). Both groups are weak bases cumulative under acidic conditions and therefore may compete for binding. Furthermore, blockage of $\mathrm{H}+, \mathrm{K}+$-ATPase by PPIs in lysosomes raises the $\mathrm{pH}$ and reduces the AD accumulation, which weakens their immunomodulatory effects. However, the in vivo effect of this potential interaction is unknown. On the other hand, it is certain that reduction of the stomach acidity leads to impaired $A D$ absorption and therefore antacids should be taken with about a 4-hour break.

There is a belief that smoking weakens the response to AD. According to several retrospective studies, smoking speeds up the AD metabolism and thereby reduces their efficacy. In addition, nicotine may inhibit lysosomal uptake of AD. However, recent studies with larger groups of patients showed no association between cigarette smoking and the efficacy and concentration of AD [10].

\section{Mechanism of action}

Antimalarials are characterized by a multidirectional effect in modifying the course of rheumatic diseases, which has not yet been fully understood. It includes an inhibitory effect on many immunological processes such as antigen presentation, cell activation, chemotaxis or inflammatory mediators. Interestingly, low affinity antigen presentation is blocked on AD treatment. In contrast, the presentation of antigens with high affinity, which include bacterial antigens, is not compromised. This may explain the observed immunomodulatory rather than immunosuppressive effect of AD, which contributes to the improved safety profile.

\section{Antimalarials and lysosomes}

The lipophilic ring in the CQ and HCQ molecules enables quick diffusion across the cell membrane into the cytoplasm, whereas weak bases accumulate in acidic organelles such as lysosomes, with even 1000-fold higher concentration. This leads to an increase in $\mathrm{pH}$ in lysosomes and decreases the activity of lysosomal enzymes. It impairs proteolysis reactions, glycation and protein secretion. Disorder of lysosomal function is one of the earliest known AD mechanisms of action and therefore is called "the lysosomotropic action or effect". Antimalarial drugs can accumulate in other cytoplasmic ultrastructures such as the Golgi apparatus and endoplasmic reticulum. The lysosomotropic effect is responsible for the proper operation of the antiparasitic effect of AD - impairment of hemoglobin degradation leads to the accumulation of heme, which is toxic to malaria parasites. In contrast, the result of abnormal proteolytic processes in the cells is inhibition of immune cell proliferation, chemotaxis, phagocytosis, production of free radicals, antigen presentation and the production of proinflammatory cytokines [9].

\section{Antimalarials and presentation of antigens}

The immunomodulatory effect of $A D$ is also apparent from the blockage of antigen presentation. Normally specialized antigen-presenting cells (APC) - dendritic cells, monocytes and macrophages - with the MHC class II molecules present foreign antigens as well as autoantigens to T cells. Antimalarials impair this process at various stages. Internal processes of antigen preparation, to connect to the MHC molecule and transport to the cell surface, are disturbed.

\section{Antimalarials and proinflammatory cytokines}

In vitro studies have demonstrated the inhibitory effect of $A D$ on proinflammatory cytokine secretion, including TNF- $\alpha$, IL-1, IL-2, IL-6, IFN- $\alpha$ and $\gamma$. These results were also reflected in vivo. In patients with SLE, CQ therapy in 3 months caused a significant reduction in serum concentrations of IL- 6, IL-18 and TNF- $\alpha$. The observed results of a multiethnic US cohort of SLE patients (LUMINA) showed significant improvement during HCQ therapy, which correlated with decreased serum IFN- $\alpha$ levels [11]. The effect observed in patients and experimental studies is caused by disruption of lysosomes and also by modulation of kinase activity in pathways involved in the production of cytokines.

\section{Antimalarial drugs and Toll-like receptors}

Another immunomodulatory mechanism of action of $A D$ is associated with inhibition of Toll-like receptors (TLRs). Toll-like receptors are proteins involved in the activation of innate immune cells, an important element in the body's first line of defense against pathogens. So far, in humans 11 TLR have been characterized, which recognize and bind many antigens: bacterial, fungal (receptors located on the surface of immune cells - TLR1, TLR2, TLR4, TLR5, TLR6, TLR10, TLR11) and viral (receptors within the endosomal membrane vesicles - TLR3, TLR7, TLR8, TLR9). As a result of the activation of TLRs, proinflammatory cytokines that can recruit other immune cells are produced, in order to ensure the effective elimination of the pathogen from the body. TLRs can also recog- 
nize self-antigens, such as nucleic acid fragments, even including those within immune complexes. In recent years, the impaired function of TLRs in the pathogenesis of SLE, RA, antiphospholipid syndrome, scleroderma and Sjögren's syndrome has been emphasized. Antimalarials suppress TLR activation, most likely by their properties of disorder of lysosomotropic receptors associated with endosomes. Another potential mechanism is the ability of AD to directly bind to nucleic acids and mask epitopes recognized by the TLR.

\section{Other potential therapeutic mechanisms of antimalarial drugs}

Other immunomodulatory effects of AD include inhibition of phospholipase $A 2$, inhibition of prostaglandin synthesis and intracellular signaling associated with the calcium ions in $T$ and $B$ cells, blockage of immune cell stimulation mitogenic factors, reduction of circulating immune complexes in the blood of RA patients, and inhibition of matrix metalloproteinases (MMPs).

\section{Use of antimalarials \\ Systemic lupus erythematosus}

The first reports of skin lesions' improvement after treatment with quinine in discoid lupus and systemic lupus erythematosus (SLE) came from the end of the nineteenth century. Currently $A D$ are used in patients with SLE with low and moderate activity, especially in the case of skin lesions, and joint and muscle involvement. The effects of AD are much wider and include reduction of fatigue, serous membrane inflammation, mouth ulcers and renal involvement. Progression of kidney damage in SLE can be halted by HCQ treatment and end-stage chronic kidney disease occurs less frequently [12]. In the latest ACR and EULAR recommendations on SLE treatment with renal involvement the use of HCQ as the primary drug was recommended $[13,14]$.

Several clinical trials have proven AD efficacy in the treatment of SLE [12]. Treatment with CQ and HCQ can lead to reduction of disease activity and concomitant lowering of doses of corticosteroids. Ruiz-Irastorza et al. [11] in a systematic review showed that AD can reduce disease activity by more than $50 \%$. Risk of organ damage in SLE is reduced by AD. According to the results of the LUMINA cohort, patients not treated with HCQ were characterized by a higher general indicator of organ damage and higher incidence of polyserositis, kidney damage, cytopenia, nervous system involvement, and more hospitalizations [10]. Further evidence for the beneficial effect of AD is increased survival of SLE patients and a reduced infection rate [11]. In addition, the use of
HCQ in the preclinical stage of SLE may delay the development of flare [12]. The retrospective study of James et al. [12] showed that patients treated with HCQ had longer disease duration from the onset to diagnosis of SLE, lower titers of antinuclear antibodies and fewer of their specifications.

According to some researchers, low HCQ concentrations in the blood of SLE patients were associated with higher disease activity and are strong predictors of flare [9]. More recent studies do not confirm these observations, but due to the large inter-individual differences in the pharmacokinetics of HCQ, only a small group of patients was able to get the drug concentration in excess of $1000 \mathrm{ng} / \mathrm{ml}$ [9].

Clearly, ceasing the use of HCQ is associated with a higher incidence of flares of SLE.

In conclusion, AD should form the basis of pharmacotherapy of SLE. It should also be emphasized that AD should not be discontinued, both in remission as well as in flare. Although they are no substitute for intensive treatment of exacerbations, it would support the treatment of severe disease.

\section{Rheumatoid arthritis}

Antimalarials have been used in the treatment of rheumatoid arthritis (RA) for about 65 years. Therapy with $A D$ reduces the number of tender and swollen joints, and reduces disease activity as assessed by the physician and the patient [2]. However, compared with other DMARDs, CQ and HCQ have a weak effect, require a relatively long time to achieve clinical improvement and did not inhibit the radiographic progression [2]. Therefore, $A D$ in the treatment of RA are an element of combination therapy. $A D$ are used in monotherapy only in mild and moderate RA or in case of intolerance of other DMARDs. According to the 2008 ACR guidelines, HCQ monotherapy is recommended in the case of low disease activity, which lasts no longer than 24 months, in the absence of bad prognostic factors. Hydroxychloroquine with sulfasalazine can be used in patients with high disease activity, without bad prognostic factors, with an average disease duration of 6-24 months. Triple therapy (methotrexate, sulfasalazine, hydroxychloroquine) is indicated for patients with RA with the presence of poor prognostic factors, with a moderate or aggressive course regardless of the duration. This combination of drugs can be a safe and effective alternative to biologics.

\section{Sjögren syndrome}

Several studies (all but one uncontrolled) that have analyzed the use of HCQ in primary Sjögren syndrome (SS) found improvement in general features (fatigue), 
glandular involvement, joint pain and laboratory abnormalities (hypergammaglobulinemia, elevation of ESR, CRP, and RF levels), and a possible benefit in reducing cardiovascular risk [14]. More than $60 \%$ of treated patients reported improvement in eye dryness, cornea integrity and ocular pain. Perhaps AD efficacy is influenced by the disease period - at the initial, inflammatory stage the symptoms can be diminished, not after damage of glands ("burned out" stage) [14].

\section{Other rheumatic diseases}

There are many reports on AD efficacy in other autoimmune diseases: palindromic rheumatism, eosinophilic fasciitis, dermatomyositis, cellulitis, mixed and undifferentiated connective tissue disease. However, in contrast to SLE and RA, evidence from controlled clinical trials is not conclusive. Most of the data on AD use are mainly based on uncontrolled studies with small groups of patients or clinical case reports.

\section{Pleiotropic action of antimalarial drugs}

The observed clinical benefits of treatment with $A D$ are not limited to the reduction of the underlying disease activity. They exhibit a number of pleiotropic effects on coagulation, lipid and carbohydrate metabolism, thus contributing to the reduction of cardiovascular risk in patients with rheumatic diseases.

\section{Carbohydrate metabolism}

The first data on the potential AD impact on carbohydrate metabolism come from 1984, when a case of a patient with diabetes mellitus treated with CQ was described and reduced need for insulin was observed [14]. Other case studies and the results of clinical trials indicate the hypoglycemic effect of AD [14]. In patients with poorly controlled type 2 diabetes therapy AD added to sulphonylureas or insulin improves glucose tolerance, provides better glycemic control and reduces glycated hemoglobin (HbA1c) levels [14]. Use of AD reduces the need for oral antidiabetics and insulin, and even completely overrides the need for insulin therapy in isolated cases [14]. In patients with rheumatic diseases and diabetes mellitus HCQ causes a significant reduction in $\mathrm{HbA}_{1}$ C. Patients with rheumatic diseases without carbohydrate disorders in their history also may benefit from the use of AD. Penn et al. [15] examined the effects of HCQ on carbohydrate metabolism in patients with RA and SLE without a history of diabetes.

It was found that HCQ lowers blood glucose in the fasting state, and in SLE patients further reduces insulin. Increased insulin sensitivity was also observed in obese patients without a history of diabetes and chronic inflammatory diseases on HCQ therapy for 6 weeks. Some reports describe the possibility of diabetes risk reduction in patients with chronic inflammatory diseases receiving HCQ [16]. In a retrospective cohort of Solomon et al. [17] 13,905 patients with RA or psoriasis showed reduced risk of developing diabetes on HCQ therapy. No other non-biologic DMARDs showed such properties. The protective effect of $A D$ on diabetes development in RA patients was also shown in other studies [16].

The exact mechanism of hypoglycemic effects in CQ and HCQ therapy is associated primarily with the impact on insulin metabolism. Antimalarials inhibit the intracellular degradation of insulin and increase insulin secretion in a dose-dependent manner by pancreatic cells [16]. The AD actions include inhibition of gluconeogenesis, prolongation of the active complex of the insulin receptor half-life in endosomes and increased glucose uptake by peripheral tissues [16].

Thanks to improvement of glycemic control in patients with rheumatic diseases, AD may reduce the increased risk of cardiovascular events. In daily practice, it must be remembered that in rare cases AD can cause serious and life-threatening hypoglycemia. Thus, it seems reasonable to control glucose values in the initial period of treatment, especially in patients with diabetes.

\section{Bone metabolism}

Prevention of osteoporosis is an important issue in pharmacotherapy of autoimmune diseases. In addition to chronic inflammation, reduced bone mineral density (BMD) can be further increased during treatment with agents such as glucocorticoids (GCS) and low molecular weight heparins. So far only a few observations suggest that use of HCQ therapy, treatment time and cumulative dose in patients with SLE correlate positively with the average spine and hip BMD [18]. Antimalarials provide protection against BMD reduction and lower the risk of osteopenia/osteoporosis, including in the group of patients who received GCS. The observed effect can be explained by a reduction in disease activity and reduced GCS doses. Lysosomotropic AD action cannot be excluded. According to recent studies a rise in the lysosomal $\mathrm{pH}$ impairs the osteoclast process of osteoclastogenesis in vitro [19].

\section{Lipid profile}

Most studies demonstrate a positive AD impact on all lipid fractions in patients with SLE and RA, even on GCS therapy. Antimalarials reduce levels of total cholesterol, low-density lipoproteins (LDL), very low-density lipoproteins (VLDL) and triglycerides (TG), and also sig- 
nificantly increase the level of high-density lipoprotein (HDL) [20]. Changes in lipid profile are detected after 3 months of AD therapy and reach a stable value after about a year of therapy. The increase of steroid-induced lipoprotein synthesis may be offset by an increase in $A D$ for $L D L$ receptor activity and accelerated the removal of lipoproteins from plasma. The mechanism of lipid changes is also apparent from a decrease in cholesterol synthesis by inhibiting HMG-CoA reductase, an enzyme which is the therapeutic point for statins - a group of medicines widely used to treat hyperlipidemia.

\section{The anticoagulant effect}

Reduction in the incidence of thromboembolic complications during AD therapy was suggested by a British orthopedist in the 1970s, when he administered HCQ as prevention of deep vein thrombosis and pulmonary embolism in patients after hip replacement surgery. Probably because of the beneficial antithrombotic effect of $A D$ it became a subject of interest in the next series of experiments.

Antithrombotic HCQ activity in SLE patients is independent from antiphospholipid antibodies' presence [21]. Jung et al. [22] observed a reduction of $66 \%$ arterial and $74 \%$ of venous thrombotic events in the group of SLE patients. Experimental studies show that HCQ inhibits platelet aggregation by blocking the surface expression of platelet activating factor-stimulated effects of antiphospholipid antibodies and thrombin agonists, as well as a decrease in the release of acetylsalicylic acid from activated platelets. Hydroxychloroquine prevents binding antiphospholipid antibodies with annexin A5 a lipid bilayer protein with strong anticoagulant properties.

HCQ may protect against thrombosis in asymptomatic antiphospholipid antibody (aPL)-positive patients. Hydroxychloroquine also reverses platelet activation, induced by aPL.

\section{Atherosclerosis}

Patients with chronic inflammatory diseases are exposed to early development of atherosclerotic lesions in blood vessels. Despite the beneficial effects on carbohydrate and reduced atherogenic lipid fractions, most studies have not demonstrated the antimalarials' inhibitory effect on the progression of atherosclerosis [12]. Only Tanay et al. [23] observed improvement in the flexibility of large vessels and a decrease in total peripheral resistance in patients with SLE under the influence of HCQ. Bengtsson et al. [24] observed improvement in the vascular vasodilator response in patients with SLE who used $A D$.

\section{Endothelium}

Antimalarials increase endothelium-dependent vasodilatation [24]. In premenopausal women with SLE there was a significantly lower prevalence of vascular stiffness treated with HCQ [25]. However, in a small study, HCQ was found to increase large artery elasticity and lowered systemic vascular resistance in SLE patients compared with those receiving no treatment or on corticosteroids alone [23].

\section{Infections}

Antimalarials were originally used only in malaria and therefore it is not too surprising that AD has shown activity in other infections. In contrast with other immunosuppressive therapies, treatment of rheumatic diseases with $A D$ has not been associated with an increased risk of infections [26]. It was confirmed in over 200 lupus nephritis patients on AD therapy [26]. The number of infections was significantly fewer in patients treated with AD [26].

In other study treatment with $A D$ was the only variable that was significantly associated with reduced risk of infections, when lung involvement and the dose of prednisone were associated with increased infection risk [27]. The postulated inhibitory effects on the growth of intracellular organisms including bacteria and viruses is alkalinization of acidic intracellular vesicles caused by AD. A similar antimicrobial effect was observed in HIV infection - decreased viral load. It is thought to be an effect of post-translational glycosylation disruption in the viral envelope protein gp120, and results in decreased infectivity of the viral particles [28]. Similar effects on other viruses including hepatitis C have been proposed [28].

\section{Malignancy}

HCQ may have antineoplastic effects. The data from one prospective study, observational cohort and clinical trials showed reduced malignant cell growth with viability and induction of cell apoptosis [29, 30]. The hazard ratio for cancer among SLE patients treated with AD compared with non-users was 0.15 (95\% Cl 0.02-0.99) [31].

\section{Problems associated with the use of antimalarial drugs}

\section{Adverse reactions}

Antimalarials are considered to be relatively safe in the treatment of rheumatic diseases. Despite their widespread use, the incidence of reported adverse reactions is low [12]. The common concern of patients and physicians is usually due to potential ocular toxicity in the cil- 
iary body and signs of retinopathy. Other AD side effects include gastrointestinal and skin symptoms [32]. Individual reports showed cardiotoxicity, myopathy, hypoglycemia, and bone marrow suppression induced by $A D$, very rarely recorded in daily clinical practice [12]. In general, doses less than $6.5 \mathrm{mg} / \mathrm{kg} /$ day for HCQ and $3 \mathrm{mg} / \mathrm{kg} /$ day for CQ are well tolerated by patients.

\section{Gastrointestinal tract}

The most frequent side effects due to $A D$ treatment from the gastrointestinal tract include decreased appetite, nausea, vomiting, diarrhea, bloating and burning sensation in the retrosternal area. The most common adverse effect is gastrointestinal upset, which may be severe enough to cause a loss of appetite. It occurs more often when using CQ (20\%) than HCQ (10\%) [33]. Antimalarials do not cause ulceration or life-threatening disorders of the gastrointestinal tract. Reported gastrointestinal symptoms may be temporary or give way when the dose is reduced. In order to improve the tolerability of the treatment dose titration and receipt of $A D$ in the evening are also used. Disorders, at least partially, are connected with the action on the smooth muscle cells of the gastrointestinal tract [32].

\section{Skin}

One of the most common side effects of AD therapy in the skin and mucous membranes is hyperpigmentation in up to $10-30 \%$ of patients receiving the drug. Pigmentation of deep violet blue-gray is located on the face, hard palate, forearms and calves. Skin lesions persist for several months after cessation of therapy [32]. The mechanism of pigmentation disorders associated with AD use is unknown. In the histopathological specimens of dermis melanin granules and hemosiderin deposits were observed. Antimalarials are likely to stimulate the melanocytes in a direct way. Skin appendages also tend to be occupied. Hair can undergo depigmentation, and on the nails transverse stripes can be formed. Hypersensitivity reactions to $A D$ can also appear. After AD dosage patients also reported itching, generalized pustular rash, urticaria and erythroderma [32]. Interestingly, a higher incidence of cutaneous intolerance was observed in patients with dermatomyositis (about 30\%) than in patients with cutaneous forms of lupus erythematosus (3-10\%) [34].

\section{Nervous system}

Nervous system disorders associated with $A D$ include headaches, nightmares, dizziness and tinnitus. These symptoms may disappear spontaneously within a few weeks or by reducing the dose. Much less fre- quently seizures, psychosis, a sense of fear or cases of mania were reported [35].

\section{Eye}

Ophthalmic adverse reactions may affect the ciliary body, cornea and retina. The most frequent are corneal deposits, which in addition to sensitivity to light in some patients may result in altered vision, such as halos or photosensitivity, but usually no change in visual acuity was reported [35]. This side-effect is rare with HCQ at a dose of $400 \mathrm{mg} /$ day and a little more common with CQ. Corneal deposits are not a contraindication to continued treatment and usually resolve 1.5-2 months after treatment is stopped [32].

Transient diplopia may occur on initiation of the therapy. Potentially the most dangerous ocular complication of chronic AD therapy is retinopathy. Initially, it manifests as red vision, loss of image of the fundus, ringed foci discoloration and the abolition of reflexes within the yellow stain. Over time, scotoma can also occur as an image called "buffalo eye" at the bottom of the eye [32]. Early toxicity may manifest with very subtle changes in the retinal pigment epithelium around the fovea. At this stage drug cessation may cause vision recovery. The classic sign - a ring of perifoveal retinal pigment layer atrophy called "bull's eye" maculopathy - is in fact a late sign. Photophobia, photopsia, significant visual field defects around central fixation and ultimately visual loss can occur at the late stage. All changes at this stage are irreversible and may even progress for a period of time following cessation of treatment [36].

The bulk of the literature on toxicity has come from patients referred because of symptoms or signs of potential retinal damage. For example, in a study of Wolfe et al. [37], 3,995 patients with RA or SLE on the HCQ treatment, among them 1,538 current users, were screened for self-reported toxicity and followed up in positive cases with detailed patient history and specialist confirmation. $6.5 \%$ of patients discontinued therapy because of an eye problem, of whom $1.8 \%$ reported HCQ retinal problems. But finally definite or probable toxicity was documented only in $0.65 \%$. The risk of toxicity was low in the initial 7 years of exposure, became 5 times greater after 7 years of usage (or 1,000 g total exposure) and was unrelated to age, weight, or daily dosage.

Due to the irreversible nature of retinopathy - in fact a very rare adverse effect - the American Association of Ophthalmology (AAO) recommended that patients at high risk once a year should have an assessment of the retina and central visual field test. Factors that increase risk include: more than five years of $A D$ exposure, doses higher than $6.5 \mathrm{mg} / \mathrm{kg} /$ day, obesity, concomitant kidney or liver disease, other diseases of the retina and age 
over 60 years. In the case of long-term treatment with standard dosages, the risk of retinopathy is $2.6 \%$ for CQ and $0.3 \%$ for $\mathrm{HCQ}$ [12].

The AAO recommends a baseline eye examination at the beginning of HCQ treatment, which will serve as a point of reference. Pre-existing macular disease will make it difficult to detect subtle early signs of toxicity and hence is a relative contraindication to antimalarial therapy. Preferred screening is annual and may begin after 5 years or sooner in older patients, those at risk of macular disease and those with pre-existing renal or liver disease. Screening tests should include biomicroscopy and an automated threshold 10-2 protocol field test. Newer objective tests should be added to the screening protocol. These tests include multifocal electroretinogram (mfERG), spectral domain optical coherence tomography (SD-OCT) and fundus autofluorescence (FAF), which can be more sensitive than visual fields in detecting the earliest changes of HCQ [38].

The Amsler grid and color vision testing are only adjunct tests and not sufficiently sensitive for detection of early HCQ retinopathy. Fundus examinations are still advised for documentation, but visible maculopathy is a late and irreversible change and should not be relied on for screening [36].

\section{Myopathy}

Antimalarial-induced myopathy has been described in the literature. These lysosomotropic CQ and HCQ effects lead to accumulation of tissue deposits inside the cells and disruption of their functions. Myopathy can manifest as progressive, painless proximal limb weakness in conjunction with diminished tendon reflexes yet with retained sensation. This effect is very difficult to distinguish from muscle involvement in systemic disease. Lactate dehydrogenase (LDH) concentration correlates with the severity of myopathy [39]. The level of creatine kinase (CK) often does not go beyond the normal range. Typically myopathy resolves rapidly after the medication is discontinued - even when this involves long-term treatment and high cumulative dosages. Neuromyopathy can also occur in a short treatment period (5-7 months) with low cumulative doses (70-80 g). The clinical improvement after discontinuation is rapid, even with high cumulative doses [39]

\section{Cardiovascular}

Although cardiovascular symptoms are very rare, they can cause dangerous complications during AD therapy. They include two groups of diseases: cardiomyopathy and arrhythmias. As in other muscles of the body, CQ and HCQ may induce cardiomyocyte dysfunction mani- fested as atrial enlargement, thickening and impaired ventricular systolic and diastolic function. Arrhythmias during treatment tend to increase slowly from bundle branch block to complete atrioventricular block [9]. QT interval prolongation and malignant ventricular arrhythmias have also been observed in individual cases [9].

Therapeutic doses cause ST-segment depression, T-wave inversion and QT interval prolongation in the resting electrocardiogram. Cardiac damage is symptomatic only at a later stage with high cumulative doses (patients with AV block: after an average of 7 years/720 g; cardiomyopathy patients: after an average of 14 years/ 1640 g). It must be noted that the cardiac process may be clinically asymptomatic for a long period and that the symptoms of toxic damage are often described as the result of underlying rheumatic disease and its complications. On the other hand, in a study of electrocardiograms (ECGs) in 85 unselected patients with connective tissue disease treated with $\mathrm{HCQ}$, the frequency of heart conduction disorders was similar to what is expected in the general population, which proves the cardiac safety of HCQ [9]. Cardiotoxicity of AD may also manifest as heart failure [36]. Toxic cardiomyopathy may be reversible if detected at an early stage, but more severe advanced cases may be refractory to cessation of $A D$, which highlights the importance of timely diagnosis [9].

\section{Cytopenias}

Very rarely cases of aplastic anemia states, agranulocytosis, leukopenia, neutropenia and thrombocytopenia have been observed [32]. In patients deficient in glucose- 6 phosphate AD are contraindicated due to the risk of hemolysis.

\section{Antimalarial drugs and pregnancy}

According to the recommendations of the expert panel published in 2006, AD can be continued in the treatment of rheumatic diseases during pregnancy and lactation (level II records) [9]. In these cases, HCQ is the drug of choice (level IV records). According to the points to consider by EULAR (European League Against Rheumatism) based on a systematic literature review and pregnancy exposure data from several registries, published in 2016, $A D$ can be continued in the treatment of rheumatic diseases during pregnancy and lactation (level II records) [9]. Although reports from preclinical studies pointed to embryo and fetotoxicity, high doses of CQ (250-1500 mg/kg) did not receive this confirmation in studies assessing the safety of therapeutic doses for human use (CQ $250 \mathrm{mg}$ ) day; HCQ 200-400 mg/day). Antimalarial concentration in maternal blood, in the placenta and in cord blood are similar. Secretion into milk is low and does not exceed 
$0.2 \mathrm{mg} / \mathrm{kg} /$ day [9]. A systematic review of the literature confirmed that HCQ treatment of patients with autoimmune diseases is not associated with an increased risk of birth defects, miscarriages, fetal death and prematurity or a reduced number of live births. Also noteworthy is the study by Clowse et al. [40], which showed a significant increase in activity and exacerbations of SLE patients held back pregnant with HCQ. In patients who continued treatment with $A D$, the average GCS dose used during pregnancy was significantly lower. In pregnant women with SLE, flares can be prevented by HCQ discontinuation [9]. In children of pregnant mothers using AD no abnormalities of sight, hearing or other organs were observed [9]. Moreover, compared to children of mothers treated with placebo, they were characterized by a higher birth weight and higher Apgar score. Pregnant patients with SLE treated with AD have reduced risk of conduction disturbances in the neonatal lupus syndrome in the case of anti-Ro/La positivity [9].

\section{Comparison of chloroquine and hydroxychloroquine}

Papers reporting the difference between CQ and $\mathrm{HCQ}$ in the medical literature are very sparse. It is estimated that the effectiveness of HCQ may be even less than half that of CQ, but with reduced toxicity and fewer adverse reactions [41]. Probably the conviction of greater CQ toxicity, particularly for retinal cells, has contributed to more frequent use of HCQ in the world. A higher frequency of $A E$ in patients receiving $C Q$ in comparison with those receiving HCQ is documented in only one study (28.4\% vs. $14.7 \%, p=0.001)$ [39]. Overall, from 15\% of patients who discontinued $A D$ due to toxicity, those receiving $\mathrm{HCQ}$ were less likely to discontinue the drug due to side effects than those taking CQ (adjusted HR $0.62,95 \% \mathrm{Cl} 0.40$ to 0.96$)$. As mentioned above, retinal toxicity was observed in $2.6 \%$ of CQ users compared to $0.3 \%$ of HCQ users [41]. The incidence of neuromyopathy appears to be lower under HCQ than under CQ, but no reliable statistical data are available [41].

\section{Conclusions}

Antimalarials still form the basis of therapies for many autoimmune diseases. The long period of AD observation, their good safety and tolerability for patients and numerous pleiotropic effects are well recognized values. Therefore, even in the era of modern immunosuppressive drugs and biologics, CQ and HCQ still occupy an important place in clinical practice of rheumatologists around the world, contributing to an increase in survival, improved quality of life and reduced risk of cardiovascular complications. Further research on the multidirectional effect of $A D$ and their mechanism of action should result in the creation of more effective and less toxic derivatives.

The authors declare no conflict of interest.

\section{References}

1. Woodward R, Doering W. The total synthesis of quinine. J Am Chem Soc 1944; 66: 849.

2. Iredale J, Fieger H, Wainer IW. Determination of the stereoisomers of hydroxychloroquine and its major metabolites in plasma and urine following a single oral administration of racemic hydroxychloroquine. Semin Arthritis Rheum 1993; 23 (Suppl. 1): 74-81.

3. Furst DE. Pharmacokinetics of hydroxychloroquine and chloroquine during treatment of rheumatic diseases. Lupus 1996; 5 (Suppl. 1): S11-S15.

4. McLachlan AJ, Tett SE, Cutler DJ, et al. Bioavailability of hydroxychloroquine tablets in patients with rheumatoid arthritis. Br J Rheumatol 1994; 33: 235-239.

5. Ono C, Yamada M, Tanaka M. Absorption, distribution and excretion of 14C-chloroquine after single oral administration in albino and pigmented rats: binding characteristics of chloroquine-related radioactivity to melanin in vivo. J Pharm Pharmacol 2003; 55: 1647-1654.

6. Tanaka E, Taniguchi A, Urano W, et al. Pharmacogenetics of disease-modifying anti-rheumatic drugs. Best Pract Res Clin Rheumatol 2004; 18: 233-247.

7. Furst DE, Lindsley $\mathrm{H}$, Baethge $\mathrm{B}$, et al. Dose-loading with hydroxychloroquine improves the rate of response in early, active rheumatoid arthritis: a randomized, double-blind six-week trial with eighteen-week extension. Arthritis Rheum 1999; 42: 357-365.

8. Costedoat-Chalumeau N, Hulot JS, Amoura Z, et al. Heart conduction disorders related to antimalarials toxicity: an analysis of electrocardiograms in 85 patients treated with hydroxychloroquine for connective tissue diseases. Rheumatology (Oxford) 2007; 46: 808-810.

9. Götestam Skorpen C, Hoeltzenbein M, Tincani A, et al. The EULAR points to consider for use of antirheumatic drugs before pregnancy, and during pregnancy and lactation. Ann Rheum Dis 2016; 75: 795-810.

10. Willis R, Seif AM, McGwin G Jr, et al. Effect of hydroxychloroquine treatment on pro-inflammatory cytokines and disease activity in SLE patients: data from LUMINA (LXXV), a multiethnic US cohort. Lupus 2012; 21: 830-835.

11. Ruiz-Irastorza G, Ramos-Casals M, Brito-Zeron P, et al. Clinical efficacy and side effects of antimalarials in systemic lupus erythematosus: a systematic review. Ann Rheum Dis 2010; 69: 2028.

12. James JA, Kim-Howard XR, Bruner BF, et al. Hydroxychloroquine sulfate treatment is associated with later onset of systemic lupus erythematosus. Lupus 2007; 16: 401-409.

13. Hahn BH, McMahon MA, Wilkinson A, et al. American College of Rheumatology guidelines for screening, treatment, and management of lupus nephritis. Arthritis Care Res (Hoboken) 2012; 64: 797-808. 
14. Gaujoux-Viala C, Smolen JS, Landewé R, et al. Current evidence for the management of rheumatoid arthritis with synthetic disease-modifying antirheumatic drugs: a systematic literature review informing the EULAR recommendations for the management of rheumatoid arthritis. Ann Rheum Dis 2010 69: 1004-1009.

15. Penn SK, Kao AH, Schott LL, et al.Hydroxychloroquine and glycemia in women with rheumatoid arthritis and systemic lupus erythematosus. J Rheumatol 2010; 37: 1136-1142.

16. Ramos-Casals M, Brito-Zerón P, Sisó-Almirall A, et al. Topical and systemic medications for the treatment of primary Sjögren's syndrome. Nat Rev Rheumatol 2012; 8: 399-411.

17.Solomon DH, Massarotti E, Garg R, et al. Association between disease-modifying antirheumatic drugs and diabetes risk in patients with rheumatoid arthritis and psoriasis. JAMA 2011; 305: 2525-2531.

18. Mok CC, Mak A, Ma KM. Bone mineral density in postmenopausal Chinese patients with systemic lupus erythematosus. Lupus 2005; 14: 106-112.

19. Voronov I, Ochotny N, Jaumouillé V, et al. The R740S mutation in the V-ATPase a3 subunit increases lysosomal pH, impairs NFATc1 translocation, and decreases in vitro osteoclastogenesis. J Bone Miner Res 2013; 28: 108-118.

20. Cairoli E, Rebella M, Danese $N$, et al. Hydroxychloroquine reduces low-density lipoprotein cholesterol levels in systemic lupus erythematosus: a longitudinal evaluation of the lipid-lowering effect. Lupus 2012; 21: 1178-1182.

21. Tektonidou MG, Laskari K, Panagiotakos DB, et al. Risk factors for thrombosis and primary thrombosis prevention in patients with systemic lupus erythematosus with or without antiphospholipid antibodies. Arthritis Rheum 2009; 61: 29-36.

22. Jung $\mathrm{H}$, Bobba $\mathrm{R}$, Su J, et al. The protective effect of antimalarial drugs on thrombovascular events in systemic lupus erythematosus. Arthritis Rheum 2010; 62: 863-868.

23. Tanay A, Leibovitz E, Frayman A, et al. Vascular elasticity of systemic lupus erythematosus patients is associated with steroids and hydroxychloroquine treatment. Ann N Y Acad Sci 2007; 1108: 24-34.

24. Bengtsson C, Andersson SE, Edvinsson L, et al. Effect of medication on microvascular vasodilatation in patients with systemic lupus erythematosus. Basic Clin Pharmacol Toxicol 2010; 107: 919-924.

25. Selzer F, Sutton-Tyrrell K, Fitzgerald S, et al. Vascular stiffness in women with systemic lupus erythematosus. Hypertension 2001; 37: 1075-1082.

26. Sisó A, Ramos-Casals M, Bové A, et al. Previous antimalarial therapy in patients diagnosed with lupus nephritis: influence on outcomes and survival. Lupus 2008; 17: 281-288.

27. Ruiz-Irastorza G, Olivares N, Ruiz-Arruza I, et al. Predictors of major infections in systemic lupus erythematosus. Arthritis Res Ther 2009; 11: R109.

28. Rahim R, Strobl JS. Hydroxychloroquine, chloroquine, and alltrans retinoic acid regulate growth, survival, an histone acetylation in breast cancer cells. Anticancer Drugs 2009; 20: 736745.

29. Savarino A, Gennero L, Chen HC, et al. Anti-HIV effects of chloroquine: mechanisms of inhibition and spectrum of activity. AIDS 2001; 15: 2221-2229.
30. Kim WU, Yoo SA, Min SY, et al. Hydroxychlor- oquine potentiates Fas-mediated apoptosis of rheumatoid synoviocytes. Clin Exp Immunol 2006; 144: 503-511.

31. Ruiz-Irastorza G, Ugarte A, Egurbide MV, et al. Antimalarials may influence the risk of malignancy in systemic lupus erythematosus. Ann Rheum Dis 2007; 66: 815-817.

32. Kalia S, Dutz JP. New concepts in antimalarial use and mode of action in dermatology. Dermatol Ther 2007; 20: 160-174.

33. Van Beek MJ, Piette WW. Antimalarials. Dermatol Clin 2001; 19: 147-160.

34. Pelle MT, Callen JP. Adverse cutaneous reactions to hydroxychloroquine are more common in patients with dermatomyositis than in patients with cutaneous lupus erythematosus. Arch Dermatol 2002; 138: 1231-1233.

35. Rynes RI. Antimalarial drugs in the treatment of rheumatological diseases. Br J Rheumatol 1997; 36: 799-805.

36. Tang C, Godfrey T, Stawell R, et al. Hydroxychloroquine in lupus: emerging evidence supporting multiple beneficial effects. Intern Med J 2012; 42: 968-978.

37. Wolfe F, Marmor MF. Rates and predictors of hydroxychloroquine retinal toxicity in patients with rheumatoid arthritis and systemic lupus erythematosus. Arthritis Care Res 2010; 62: 775-784.

38. Missner S, Kellner U. Comparison of different screening methods for chloroquine/hydroxychloroquine retinopathy: multifocal electroretinography, color vision, perimetry, ophthalmoscopy, and fluorescein angiography. Graefes Arch Clin Exp Ophthalmol 2012; 250: 319-325.

39. Casado E, Gratacós J, Tolosa C, et al. Antimalarial myopathy: an underdiagnosed complication? Prospective longitudinal study of 119 patients. Ann Rheum Dis 2006; 65: 385-390.

40. Clowse ME, Magder L, Witter F. Hydroxychloroquine in lupus pregnancy. Arthritis Rheum 2006; 54: 3640-3647.

41. Aviña-Zubieta JA, Galindo-Rodriguez G, et al. Long-term effectiveness of antimalarial drugs in rheumatic diseases. Ann Rheum Dis 1998; 57: 582-587. 